the summary of the baseline data. The "zero concentration" or ad iib water intake point represents a mean of 12 animals carried on free drinking prior to the alcohol ingestion tests. The points for the alcohol concentrations of $6 \%, 12 \%$, and $24 \%$ are based on means for 6 animals in each group on the last test day. The alcohol intake data were relatively stable from day to day, and all animals, including those in the $24 \%$ alcohol group continued to gain weight throughout the testing sessions. Food consumption dropped on alcohol days, as did urinary volume, in amounts roughly proportional to the concentration. On alternate "water days" fluid intake was increased in proportion to the alcohol concentration offered on the "alcohol days" and the urinary volume was similarly affected. Large individual differences in amount of alcohol accepted were noted for all concentrations (indicated by the range brackets in the figure) as considerable overlap between concentrations occurred.

An effective polydipsia as a result of the median eminence lesions occurred in 5 of the 12 animals submitted to surgery. A criterion of 2 successive days with a minimum of $100 \mathrm{cc}$ of water consumption within the 10-day postoperative period was used to define diabetes insipidus. Animals failing to meet this criterion were not included in this report. The upper tracing of the figure shows the means of these five animals tested successively with each of the three concentrations. Repeated measures were made with the $6 \%$ and the $24 \%$ concentrations, but a single measure was made for the $12 \%$ concentration. All lesioned animals showed rapid weight loss during this period, but only one death occurred (not a criterion animal). Pronounced polydipsia was noted for all animals on "water days."

It can be seen from the figure that an increase in fluid ingestion as a result of diabetes insipidus occurred only in the case of water and $6 \%$ ethanol $(\mathrm{P}=.001$ and .002 , respectively, by Mann-Whitney U test). Differences were not observed in pre- and postoperative fluid intake with the $12 \%$ and $24 \%$ alcohol concentrations ( $\mathrm{P}=.396$ and .268 , respectively). No concentration sequence effects were noted.

The alternate-day presentation schedule proved to be an effective baseline procedure, and the results approximate those functions reported by other investigators (Richter \& Campbell, 1940; Holman \& Myers, 1968). The failure of the polydipsia resulting from median eminence lesions to increase any but alcohol concentrations within the preferred range is in keeping with those findings of Holman \& Myers (1968) in which schedule-induced polydipsia was utilized, but not with those data of Cicero \& Myers (1969) in which drug-induced polydipsia actually depressed alcohol appetite. Within the range of concentrations used here, no depression in intake was noted up to the $24 \%$ upper value.

\section{REFERENCES}

CICERO, T. J., \& MYERS, R. D. Preference-aversion functions for alcohol after cholinergic stimulation of the brain and fluid deprivation. Physiology \& Behavior, 1969, 4, 559-562.

CROW, L. T. Diencephalic influence in alcohol diuresis. Physiology \& Behavior. $1968,3,319-322$

HOLMAN, B. R., \& MYERS, R. D. Ethanol consumption under conditions of psychogenic polydipsia. Physiology \& Behavior, 1968, 3, 369-371.

RICHTER, C. P., \& CAMPBELL, K. $H$. Alcohol taste thresholds and concentrations of solutions preferred by rats. Science, 1940,91,507-508.

RICK, J. T., \& WILSON, C. W. Alcohol preference in the rat. Quarterly Journal of Studies on Alcohol, 1966, 27, 447-458.

van DYKE, H. B., \& AMES, R. G. Alcohol diuresis. Acta Endocrinology, 1951, 7. 110-121.

\title{
The relationship between EEG amplitude and the rate of classical conditioning
}

\author{
HIDEO ASATO and DONOVAN E. FLEMING \\ Veterans Administration Hospital, Phoenix, Ariz. 85012
}

Six cats were used as $\mathrm{Ss}$ in a classical conditioning study to determine the rate of conditioning when the conditioned stimulus was presented during bursts of EEG activity which exceeded 80 microvolts (experimental group) and 20 microvolts (control group). It was observed that the animals conditioned significantly faster under the high-amplitude conditions than under the low-amplitude conditionis. These results were related to differences in cortical excitability during high-and low-amplitude EEG activity.

A considerable amount of effort has been expended in the description of electroencephalographic (EEG) activity following the presentation of a meaningful stimulus (see Morrell, 1961, for review). On the other hand, there is a paueity of information concerning the behavioral consequences of presentation of a stimulus during periods of systematically defined electrocortical activity which precede and are ongoing at the instant of stimulus presentation (Beck, Doty, \& Kooi, 1958). Accordingly, the present investigation was designed to examine the rate of acquisition of a conditioned flexion response (CR) when the conditioned stimulus (CS) was presented during bursts of electrocortical activity which exceeded a defined amplitude.

\section{SUBJECTS}

Six adult male cats served as the Ss 
of this investigation; these were separated into control and experimental groups comprising three cats each.

\section{APPARATUS}

The experiment was carried out in a sound-reduced electrically shielded room. The cat was placed in a canvas hammock and held with light restraint which allowed free movement of head and limbs. Brain activity was amplified and graphically written by a Grass Model 7 polygraph with capacitance-coupled EEG preamplifiers (frequency response, 0.3 to $3,000 \mathrm{~Hz}$ ). One channel of EEG activity was led into a Schmitt trigger which controlled the conditioning trial sequence through BRS-Foringer programming equipment. The CS was a buzzer presented at approximately $100 \mathrm{~dB}$ above the room ambient sound-pressure level. The unconditioned stimulus (US) was a brief 1-mA shock presented to the right hindleg. The CS-US interval was 1 sec. An ENSCO function generator was used to calibrate the Schmitt trigger

\section{PROCEDURE}

The Ss were surgically prepared with bilateral indwelling electrodes overlying the marginal and midectosylvian gyri. Electrodes for reference and grounding were placed in the occipital crest and frontal sinus, respectively. Following recovery, the animals were acclimatized to the hammock and were then habituated to the CS. At the conclusion of the habituation period, viz, the habituation of orientation responses to the $\mathrm{CS}$, the conditioning procedure was carried out. The Ss received 25 conditioning trials per day with a 30 to $90-\mathrm{sec}$ intertrial interval. The criterion of conditioning was set at $90 \%$ CRs within a daily session of 25 trials. The conditioning sequence for the experimental group was activated by EEG amplitudes which exceeded 80 microvolts. For the control group the CS-US sequence was initiated by any EEG voltage levels that exceeded 20 microvolts. The onset of the CS was made contingent upon the voltage level of the EEG monitored from the cat auditory cortex (midectosylvian gyrus). The trigger pulse was initiated on the ascending phase of a negative electrocortical wave. Essentially, the design of the experiment was to examine the rate of conditioning when the CS was presented during high-voltage EEG vs EEG activity of random voltage. The CR was monitored from an electromyographic (EMG) lead attached to the right hindleg of the $S$ in the experimental situation. When the conditioning criterion had been reached, an extinction procedure was carried out.

\section{RESULTS}

A repeated coupling of the US with the buzzer CS produced a generalized arousal (desynchronized EEG activity) in the Ss, such that for the most part the electrocortical pattern was characterized by low-voltage fast-frequency (LVF) waves with occasional bursts of high-amplitude waves (HVS). These high-amplitude waves were generally of a slower frequency than the LVF pattern, indicating a synchronized cortical discharge. Accordingly, there was an implicit correlation between the voltage and the synchrony of EEG patterns used as variables for the two groups of Ss. The high-amplitude waves exclusively triggered the CS-US sequence for the experimental animals, while high-voltage waves triggered the CS-US sequence on approximately $10 \%$ of the trials for the control animals. With either group, the onset of the buzzer produced a transition from HVS to LVF patterns.

The experimental conditions produced a marked difference in rate of CR acquisition for the two groups of Ss. The control animals reached conditioning criterion in a mean number of 640 trials $(148,849$, and 924 trials, respectively, for the three control cats). On the other hand, the experimental group reached criterion in a mean number of 72 trials $(49,69$, and 98 trials for the three experimental cats). The difference between groups was statistically reliable $(p<.05$, Wilcoxon test for unpaired data). While the groups did differentiate in rate of acquisition, the two groups were not different in rate of extinction $(p>.50)$.

\section{DISCUSSION}

Electrocortical desynchronization generally has been noted to be a concomitant of the acquisition of a behavioral response (Morrell, 1961). So also, in the present study, the onset of the $C S$ resulted in the production of a desynchronized EEG pattern provided that the preexisting EEG pattern was not desynchronized when the CS was presented to the S. With the presentation of the CS consistently in HVS EEG activity, as opposed to the random presentation in predominantly LVF EEG activity, a marked effect on the rate of acquisition of the flexion $\mathrm{CR}$ also was observed. There was no overlap in the distribution of trials-to-criterion scores for the two groups of Ss. These differences in acquisition rates presumably can be attributed to differences in cortical excitability. In a comparison of evoked potential amplitude during control and conditioning situations, Pickenhain \& Klingberg (1965) noted that during early conditioning an excessive nonspecilic electrocortical desynchronization was accompanied by evoked potentials of diminished amplitude as compared to the control responses. It is suggested by these authors that desynchronization of cortical neural activity results in a diminished number of cortical units available for evoked discharge. Thus the number of neurons available for evoked discharge would be related to the degree of cortical synchrony. According to Bishop (1958), the negative phase of synchronized cortical discharge is associated with an increase in cellular excitability to afferent stimuli. Neurons subliminal to the incoming radiation discharges alone are facilitated by the excitable phase of the neuron's intrinsic thythm. Since with both groups of animals the CS was triggered by an ascending negative wave, the onset of the CS was presented during an increasing phase of cortical excitability. However, with synchronized EEG activity, the afferent input from the $\mathrm{CS}$ would stimulate a greater number of cortical units than would an afferent discharge projected to the cortex during desynchronized EEG activity. Therefore, the presentation of the CS to the experimental animals would be more effective than would the CS presentation to control animals, as a result of the differences in cortical excitability at the time the CS was presented. Presumably, this would be the basis for differences in rate of conditioning in the present study. Dustman \& Beck (1965) and Lansing (1957) have shown a definite facilitation of behavioral performance when stimuli have been presented during the negative phase of synchronized human electrocortical activity.

\section{REFERENCES}

BECK, E C DOTY $R$, W \& KOOI $K$ A Electrocortical reactions associated with conditioned flexion reflexes. Electroencephalography \& Clinical Neurophysiology, 1958, 10, 279-289.

BISHOP, $G$. $H$. The dendrite: Receptive pole of the neurone. Electroencephalography \& Clinical Neurophysiology, 1958, Supplement 10 , 12-21.

DUSTMAN, R. E.. \& BECK, E. C. Phase of alpha brain waves, reaction time and visually evoked potentials Electroencephalography \& Clinical Neurophysiology, $1965,18,433-440$.

LANSING, $R$. W. Relation of brain and tremor rhythms to visual reaction time. Electroencephalography \& Clinical Neurophysiology, $1957,9,497-504$.

MORRELL, F. Electrophysiological contributions to the neural basis of learning. Physiological Reviews, 1961, 41. 443-494.

PICKENHAIN, L., \& KLINGBERG, F. Behavioural and electrophysiological changes during avoidance conditioning to light flashes in the rat Electroencephalography \& Clinical Neurophysiology, $1965,18,464-476$. 